\title{
CONCEPTUALIZACIÓN Y CARACTERÍSTICAS SOCIODEMOGRÁFICAS/CLÍNICAS DE LAS PERSONAS CON VIH QUE RESIDEN EN CENTROS RESIDENCIALES
}

\author{
Ralf Pascual Marijuan \\ Universidad de Burgos (España) \\ ralf@sidaburgos.org \\ Må Isabel García Alonso \\ Universidad de Burgos (España)
}

Fecha de Recepción: 24 Enero 2019

Fecha de Admisión: 30 Abril 2019

\section{RESUMEN}

Dentro de las 150000 Personas con VIH (PVIH) que se estima que hay actualmente en España, hay un colectivo de PVIH que residen en centros residenciales, por carecer de las capacidades necesarias para afrontar las exigencias del funcionamiento diario. No obstante, no hay una definición clara y poco se sabe de las características sociodemográficas y clínicas de este colectivo. Se llevó a cabo una revisión bibliográfica sobre las PVIH que residen en centros residenciales, y se administró una ficha de datos sociodemográficos y clínicos a $60 \mathrm{PVIH}$ que residen en centros residenciales y a 60 PVIH con vivienda estable, comparándose dichas características entre ambos grupos mediante la prueba exacta de Fisher, la prueba chi de los cuadrados y la prueba t Student. Se propone el término de PVIH en situación de vulnerabilidad social, como una conceptualización que reconoce la potencionalidad y capacidad de aprendizaje de este colectivo de PVIH. Las PVIH en situación de vulnerabilidad social muestran menores recuentos de linfocitos T-CD4 $\left(X^{2} \leq .05\right)$, son mayoritariamente hombres $(F \leq .05)$, presentan un menor nivel de estudios $\left(X^{2} \leq .05\right)$, la gran mayoría están desempleadas $(F \leq .05)$, participan en menor medida en voluntariados $(F \leq .05)$ y presentan una mayor asistencia a cursos o clases $(F \leq .05)$. Los hallazgos sugieren que las PVIH en situación de vulnerabilidad social muestran unas características propias, que son el punto de partida para el desarrollo de intervenciones socioeducativas que mejoren su Calidad de Vida.

Palabras clave: persona con VIH; vulnerabilidad social; centro residencial

\section{ABSTRACT}

Conceptualization and sociodemographic/clinical characteristics of people with hiv residing in residential centers. Among the 150000 people with HIV (PHIV) estimated to be currently in Spain, there is a group of PHIV residing in residential centers, because they lack the necessary skills to meet the demands of daily functioning. However, there is no clear definition and little is known 


\section{CONCEPTUALIZACIÓN Y CARACTERÍSTICAS SOCIODEMOGRÁFICAS/CLÍNICAS DE LAS PERSONAS CON VIH QUE RESIDEN EN CENTROS RESIDENCIALES}

about the sociodemographic and clinical characteristics of this group. A literature review was carried out on PHIV residing in residential centers, and a sociodemographic and clinical data sheet was administered to 60 PHIV residing in residential centers and 60 PHIV with stable housing, comparing these characteristics between both groups by Fisher's exact test, the chi test of the squares and the Student $t$ test. The term of PHIV in situation of social vulnerability is proposed, as a conceptualization that recognizes the power and learning capacity of this group of PHIV. PHIV in situation of social vulnerability show lower T-CD4 lymphocyte counts $(X 2 \leq .05)$, are mostly men $(F \leq .05)$, have a lower level of education ( $\mathrm{X} 2 \leq .05)$, the vast majority are unemployed $(\mathrm{F} \leq .05)$, participate to a lesser extent in volunteering $(F \leq .05)$ and present a greater attendance to courses or classes $(F \leq .05)$. The findings suggest that PHIV in situation of social vulnerability show their own characteristics, which are the starting point for the development of socio-educational interventions that improve their quality of life.

Keywords: people with HIV; social vulnerability; residential center

\section{ANTECEDENTES DE LAS PERSONAS CON VIH QUE RESIDEN EN CENTROS RESIDENCIALES}

Los últimos datos disponibles de ONUSIDA (UNAIDS, 2018) estiman que actualmente hay 36.9 millones (entre 31.1 y 43.9 millones) de Personas con VIH (PVIH), que en el 2017 hubo 1.8 millones (entre 1.4 y 2.4 millones) de nuevos adultos y niños infectados por VIH y que unas 940000 (entre 670000 y 1.3 millones) de personas murieron a causa de enfermedades relacionadas con el SIDA en todo el mundo (Tabla 1).

Tabla 1

Estadísticas y características regionales del VIH y SIDA.

\begin{tabular}{|c|c|c|c|}
\hline & $\begin{array}{c}\text { Adultos y niños } \\
\text { con VIH }\end{array}$ & $\begin{array}{c}\text { Nuevos adultos } \\
\text { y niños } \\
\text { infectados con } \\
\text { VIH }\end{array}$ & $\begin{array}{c}\text { Adultos y niños } \\
\text { muertos } \\
\text { debidos al } \\
\text { SIDA }\end{array}$ \\
\hline $\begin{array}{l}\text { África Oriental y } \\
\text { Meridional }\end{array}$ & $\begin{array}{l}19.6 \text { millones } \\
(17.5-22.0 \\
\text { millones })\end{array}$ & $\begin{array}{c}800000 \\
(650000-1.0 \\
\text { millón })\end{array}$ & $\begin{array}{c}380000 \\
(300000- \\
510000)\end{array}$ \\
\hline $\begin{array}{l}\text { África Occidental y } \\
\text { Central }\end{array}$ & $\begin{array}{l}6.1 \text { millones } \\
\text { (4.4-8.1 } \\
\text { millones })\end{array}$ & $\begin{array}{l}370000 \\
(220000- \\
570000)\end{array}$ & $\begin{array}{l}280000 \\
(180000- \\
410000)\end{array}$ \\
\hline $\begin{array}{l}\text { Oriente Medio y Norte de } \\
\text { África }\end{array}$ & $\begin{array}{c}220000 \\
(150000- \\
300000)\end{array}$ & $\begin{array}{c}18000 \\
(10000-31000)\end{array}$ & $\begin{array}{c}9800 \\
(6400-15000)\end{array}$ \\
\hline Asia y el Pacífico & $\begin{array}{l}5.2 \text { millones } \\
(4.1-6.7 \\
\text { millones) }\end{array}$ & $\begin{array}{c}280000 \\
(210000- \\
390000)\end{array}$ & $\begin{array}{l}170000 \\
(110000- \\
280000)\end{array}$ \\
\hline América Latina & $\begin{array}{l}1.8 \text { millones } \\
(1.5-2.3 \\
\text { millones })\end{array}$ & $\begin{array}{l}100000 \\
(77000- \\
130000)\end{array}$ & $\begin{array}{c}37000 \\
(26000-51000)\end{array}$ \\
\hline Caribe & $\begin{array}{l}310000 \\
(260000- \\
420000)\end{array}$ & $\begin{array}{c}15000 \\
(11000-26000)\end{array}$ & $\begin{array}{c}10000 \\
(7100-17000)\end{array}$ \\
\hline $\begin{array}{l}\text { Europa Oriental y Asia } \\
\text { Central }\end{array}$ & $\begin{array}{l}1.4 \text { millones } \\
(1.3-1.6 \\
\text { millones })\end{array}$ & $\begin{array}{c}130000 \\
(120000- \\
150000)\end{array}$ & $\begin{array}{c}34000 \\
(25000-41000)\end{array}$ \\
\hline $\begin{array}{l}\text { América del Norte y } \\
\text { Europa Occidental y } \\
\text { Central }\end{array}$ & $\begin{array}{l}2.2 \text { millones } \\
(1.9-2.4 \\
\text { millones })\end{array}$ & $\begin{array}{c}70000 \\
(57000-84000)\end{array}$ & $\begin{array}{c}13000 \\
(9900-18000)\end{array}$ \\
\hline TOTAL & $\begin{array}{l}36.9 \text { millones } \\
\text { (31.1-43.9 } \\
\text { millones) }\end{array}$ & $\begin{array}{l}1.8 \text { millones } \\
(1.4-2.4 \\
\text { millones })\end{array}$ & $\begin{array}{c}940000 \\
(670000-1.3 \\
\text { millones })\end{array}$ \\
\hline
\end{tabular}


En España se calcula que hay 150000 PVIH (entre 140000 y 170000), de las cuales 120000 tienen acceso al TAR (UNAIDS, 2018).

Según el Área de Vigilancia de VIH y Comportamientos de Riesgo (2018), en el año 2017 hubo 3381 personas que se infectaron por el VIH y 571 casos de personas en fase SIDA (Síndrome de Inmunodeficiencia Adquirida).

En el año 2017, en Castilla y León se notificaron 91 nuevos casos de infección por VIH, según el último informe de Vigilancia Epidemiológica de Nuevas Infecciones por VIH del Servicio de Epidemiología de la Junta de Castilla y León (2017), los cuales se distribuyen de forma dispar en las diferentes provincias (Tabla 2).

Tabla 2

Cifras de nuevas infecciones por VIH en Castilla y León en el año 2017

\begin{tabular}{cccccccccc}
\hline $\begin{array}{c}\text { Ávil } \\
\text { a }\end{array}$ & $\begin{array}{c}\text { Burgo } \\
\text { s }\end{array}$ & $\begin{array}{c}\text { Leó } \\
\mathbf{n}\end{array}$ & $\begin{array}{c}\text { Palenci } \\
\mathbf{a}\end{array}$ & $\begin{array}{c}\text { Salaman } \\
\text { ca }\end{array}$ & $\begin{array}{c}\text { Segovi } \\
\mathbf{a}\end{array}$ & $\begin{array}{c}\text { Sori } \\
\mathbf{a}\end{array}$ & $\begin{array}{c}\text { Valladol } \\
\text { id }\end{array}$ & $\begin{array}{c}\text { Zamor } \\
\mathbf{a}\end{array}$ & $\begin{array}{c}\text { CY } \\
\text { L }\end{array}$ \\
\hline 2 & 22 & 13 & 5 & 16 & 5 & 3 & 22 & 3 & 91
\end{tabular}

Fuente: Servicio de Epidemiologia de la Junta de Castilla y León (2017).

Hay diferentes estudios que hablan que las PVIH presentan en general problemas en el acceso y en el mantenimiento del empleo (Kass, Muñoz, Chen, Zucconi y Bing, 1994; Erlandson et al., 2012; Blackstone et al., 2013; Miners et al., 2014), problemas financieros (Fuster-RuizdeApodaca et al., 2018), problemas de estigma relacionado con el VIH (Fuster-RuizdeApodaca, Molero, Holgado y Ubillos, 2015), una mayor prevalencia de factores de riesgo para la salud como el consumo de tabaco, la obesidad o el abuso de sustancias tóxicas (Deeks, 2011) y un desarrollo prematuro de enfermedades asociadas al envejecimiento (Guaraldi et al., 2011).

Dentro de las PVIH, hay un colectivo que precisan de residir en centros residenciales, cuando los problemas descritos anteriormente, afectan de forma significativa a su capacidad de afrontar las exigencias del funcionamiento diario. Peña y Álvarez (1998) ya destacaban que hay un subgrupo de PVIH en estado avanzado que carecen de apoyo familiar y con dificultades económicas para afrontar su situación, las cuales precisan de un lugar de acogida que les cubra las necesidades de vivienda, alimentación, vestido, atención y apoyo psicosocial, hasta que sea posible su reintegración en la familia de origen, la derivación a otro recurso o se establezca la casa como núcleo residencial.

Se han propuesto diversos términos para referirse a este colectivo de PVIH, como Jaquotot et al. (2002) que hablan de pacientes VIH con problemática psicosocial, como aquellas PVIH con situaciones extremas como trastornos psiquiátricos, adicción de drogas complejas, reclusión, inmigración o marginalidad extrema. Otros autores acuñaron el término de SIDA social para referirse a las PVIH con problemas de marginalidad grave, que presentan una serie de características comunes: adquieren la infección por VIH por vía parenteral, se encuentran en situación de marginación y exclusión, tienen un precario o nulo apoyo socio-familiar, padecen graves infecciones y tumores oportunistas, presentan déficits neurocognitivos, frecuente necesidad de hospitalización, no siguen revisiones e incumplen el TAR. En definitiva, destacan que son PVIH que cuando se le plantean el alta hospitalaria, presentan un deterioro físico y/o mental que le condiciona de forma parcial o absoluta, lo que dificulta 0 imposibilita la supervivencia individual (Vergara-Moragues, de Campos y GirónGonzález, 2003).

Atendiendo al Comité Ciudadano AntiSida de Burgos (CCASBU, 2017), se habla de que hay un colectivo de PVIH que precisan de residir en centros residenciales, encontrándonos con diferentes perfiles de PVIH residentes, según las características que presenten: 


\section{CONCEPTUALIZACIÓN Y CARACTERÍSTICAS SOCIODEMOGRÁFICAS/CLÍNICAS DE LAS PERSONAS CON VIH QUE RESIDEN EN CENTROS RESIDENCIALES}

No responden bien a los tratamientos actuales o se encuentran fases avanzadas de la infección por VIH.

Presentan un diagnóstico tardío en situación de rescate.

Combinan problemas relacionados con el VIH/SIDA con otros problemas de salud, enfermedad 0 discapacidad (física, sensorial, psicológica y/o intelectual).

Se encuentran en situación de exclusión social y/o en situación irregular.

Tienen un precario o nulo apoyo socio-familiar.

Presentan reducidos o nulos recursos económicos y/o con recursos personales muy deteriorados o mermados.

Se encuentran con un escaso o nulo apoyo o atención en los recursos ordinarios.

No obstante, no hay una definición consensuada de este colectivo de PVIH, ya que cada autor lo denomina de una forma, atendiendo a las características que presentan estas PVIH, pudiendo ser la mayoría de estas características negativas o estigmatizadoras. Por otra parte, poco se sabe de las características sociodemográficas y clínicas de este colectivo, habiendo escasos estudios y encontrándose dicha información fragmentada en los informes técnicos que desarrolla cada centro residencial.

\section{OBJETIVOS DE LA INVESTIGACIÓN}

Describir las características sociales, demográficas y clínicas de las PVIH que residen en centros residenciales.

Desarrollar una conceptualización de las PVIH que residen en centros residenciales, que reconozca la potencialidad y capacidad de aprendizaje de este colectivo.

\section{MUESTRA}

Atendiendo al Instituto Nacional de Estadística (INE) en el año 2016 los centros de alojamiento para personas sin hogar en España acogieron a 16437 personas diariamente, representando el 5.3\% de las situaciones atendidas por parte de estos centros las relacionadas con la infección por VIH, por lo que se puede estimar que los centros de alojamiento para personas sin hogar orientados prioritariamente a la infección por VIH acogieron diariamente a unas 871 PVIH en el 2016, las cuales podrían representar a la población de PVIH que precisan residir en centros residenciales en España (INE, 2017). La muestra del estudio se seleccionó por muestreo no probabilístico, por conveniencia, participando 120 PVIH mayores de 18 años, diagnosticadas por médicos especialistas por medio de la técnica ELISA (Ensayo por Inmunoabsorción Ligado a Enzimas) y confirmado por la técnica Western Blot. La muestra se distribuyó en 2 grupos: un primer grupo formado por $60 \mathrm{PVIH}$ que residían, en el momento del estudio, en centros residenciales y un segundo grupo formado por 60 PVIH que tenían una situación de vivienda estable.

Los criterios de exclusión fueron: ser menor de edad, incapacidad para leer y/o escribir casteIlano, no tener una adherencia al TAR de al menos 2 meses y presentar tales déficits cognitivos, sensoriales 0 motores que les impidiese cumplimentar la ficha de datos sociodemográficos 0 dar el consentimiento informado de forma adecuada.

\section{METODOLOGÍA}

Hay dos diseños de investigación. El primero es de carácter descriptivo, con el fin de describir las características sociales, demográficas y clínicas de las PVIH que residen en centros residenciales. Se compararon las características entre ambos grupos mediante la prueba exacta de Fisher, la prueba chi de los cuadrados y la prueba t Student.

Y el segundo diseño se basó en una amplia búsqueda de referencias bibliográfica sobre las PVIH 
que residen en centros residenciales, con el asesoramiento de profesionales especializados en el VIH, como la del presidente del Comité Ciudadano Antisida de Burgos, y el de la directora y la psicóloga del centro residencial para PVIH La Encina.

\section{INSTRUMENTOS UTILIZADOS}

Se pasó una ficha de datos sociodemográficos y clínicos a los dos grupos de PVIH, la cual contiene la presentación y explicación del estudio, el formulario de consentimiento informado y los diferentes ítems demográficos (provincia, nacionalidad, edad, sexo, género), sociales (nivel de estudios, situación laboral, participación en voluntariado, labores del hogar y en cursos/clases) y clínicos (fecha de diagnóstico del VIH, adherencia actual al TAR, nivel de carga viral y recuento de linfocitos T-CD4). Al grupo de PVIH en centros residenciales además, se les paso ítems extras de carácter social (dependencia a sustancias, tiempo viviendo en un centro residencial y cumplimiento de las normas de convivencia del centro residencial) y clínico (año que empezó a tomarse el TAR, nadir CD4, existencia de lesión neurológica y toma de medicación psiquiátrica).

\section{RESULTADOS ALCANZADOS}

Con respecto al primero objetivo, los resultados se presentan en la Tabla 3.

Tabla 3

Características sociodemográficas y clínicas de la muestra $(N=120)$

\begin{tabular}{|c|c|c|c|c|c|}
\hline $\begin{array}{l}\text { Característic } \\
\text { as }\end{array}$ & $\begin{array}{l}\text { PVIH en centro } \\
\text { residen } \\
60\end{array}$ & $\mathbf{N}=$ & $\begin{array}{r}\text { PVIH con v } \\
\text { est }\end{array}$ & $\mathrm{J}=60$ & $\begin{array}{l}\text { Prueba } \\
\text { de } \\
\text { significaci } \\
\text { ón }\end{array}$ \\
\hline Nacionalidad & $\begin{array}{l}\text { Española } \\
\text { Extranjera }\end{array}$ & $\begin{array}{l}88.3 \\
11.7\end{array}$ & $\begin{array}{l}\text { Española } \\
\text { Extranjera }\end{array}$ & $\begin{array}{l}86.7 \\
13.3\end{array}$ & $\mathrm{~F}>.05$ \\
\hline Edad & $\begin{array}{l}\bar{x} \\
\sigma \\
\text { Rango }\end{array}$ & $\begin{array}{c}52.2 \\
1 \\
7.38 \\
37-84\end{array}$ & $\begin{array}{l}\bar{x} \\
\sigma \\
\text { Rango }\end{array}$ & $\begin{array}{c}51,9 \\
1 \\
7.03 \\
24-64\end{array}$ & $\mathrm{p}>.05$ \\
\hline Sexo & $\begin{array}{l}\text { Hombre } \\
\text { Mujer }\end{array}$ & $\begin{array}{l}76.7 \\
23.3\end{array}$ & $\begin{array}{l}\text { Hombre } \\
\text { Mujer }\end{array}$ & $\begin{array}{l}58.3 \\
41.7\end{array}$ & $\mathrm{~F} \leq .05$ \\
\hline Género & $\begin{array}{l}\text { Hombre } \\
\text { Mujer }\end{array}$ & $\begin{array}{l}76.7 \\
23.3\end{array}$ & $\begin{array}{l}\text { Hombre } \\
\text { Mujer }\end{array}$ & $\begin{array}{l}58.3 \\
41.7\end{array}$ & $\mathrm{~F} \leq .05$ \\
\hline Estudios & $\begin{array}{l}\text { Sin completar } \\
\text { estudios } \\
\text { básicos } \\
\text { GE o EGB } \\
\text { ESO o FP1 } \\
\text { FP2, BACH o } \\
\text { estudios } \\
\text { universitarios }\end{array}$ & $\begin{array}{r}50.0 \\
3.3 \\
8.3\end{array}$ & $\begin{array}{l}\text { Sin completar } \\
\text { estudios } \\
\text { básicos } \\
\text { GE o EGB } \\
\text { ESO o FP1 } \\
\text { FP2, BACH o } \\
\text { estudios } \\
\text { universitarios }\end{array}$ & $\begin{array}{l}30.0 \\
16.7 \\
36.7\end{array}$ & $\begin{array}{c}X^{2} \leq \\
.05\end{array}$ \\
\hline Trabajo & $\begin{array}{l}\text { Con trabajo } \\
\text { Sin trabajo }\end{array}$ & $\begin{array}{r}3.3 \\
96.7\end{array}$ & $\begin{array}{l}\text { Con trabajo } \\
\text { Sin trabajo }\end{array}$ & $\begin{array}{l}48.3 \\
51.7\end{array}$ & $\mathrm{~F} \leq .05$ \\
\hline Voluntariado & $\begin{array}{l}\text { Voluntario } \\
\text { No voluntario }\end{array}$ & $\begin{array}{l}11.7 \\
88.3\end{array}$ & $\begin{array}{l}\text { Voluntario } \\
\text { No voluntario }\end{array}$ & $\begin{array}{l}40.0 \\
60.0\end{array}$ & $\mathrm{~F} \leq .05$ \\
\hline $\begin{array}{l}\text { Labores del } \\
\text { hogar }\end{array}$ & $\begin{array}{l}\text { Participa } \\
\text { No participa }\end{array}$ & $\begin{array}{l}81.7 \\
18.3\end{array}$ & $\begin{array}{l}\text { Participa } \\
\text { No participa }\end{array}$ & $\begin{array}{l}90.0 \\
10.0\end{array}$ & $F>.05$ \\
\hline Cursos/Clases & $\begin{array}{l}\text { Asiste } \\
\text { No asiste }\end{array}$ & $\begin{array}{l}48.3 \\
51.7\end{array}$ & $\begin{array}{l}\text { Asiste } \\
\text { No asiste }\end{array}$ & $\begin{array}{l}18.3 \\
81.7\end{array}$ & $\mathrm{~F} \leq .05$ \\
\hline $\begin{array}{l}\text { Dependencia } \\
\text { a } \\
\text { sustancias }\end{array}$ & $\begin{array}{l}\text { No, nunca } \\
\text { Si, más de } 1 \text { año } \\
\text { Si, en el último año }\end{array}$ & $\begin{array}{l}21.7 \\
55.0 \\
23.3\end{array}$ & & & \\
\hline Meses en un & $\bar{x}$ & $\begin{array}{c}52.0 \\
8\end{array}$ & & & \\
\hline
\end{tabular}




\section{CONCEPTUALIZACIÓN Y CARACTERÍSTICAS SOCIODEMOGRÁFICAS/CLÍNICAS DE LAS PERSONAS CON VIH QUE RESIDEN EN CENTROS RESIDENCIALES}

\begin{tabular}{|c|c|c|c|c|c|}
\hline centro & Rango & $\begin{array}{c}2- \\
288\end{array}$ & & & \\
\hline Normas de & No cumple & 50.0 & & & \\
\hline convivencia & Si cumple & 50.0 & & & \\
\hline $\begin{array}{l}\text { Diagnóstico } \\
\text { del }\end{array}$ & Antes de 1996 & 48.3 & Antes de 1996 & 56.7 & $\mathrm{~F}>.05$ \\
\hline VIH & Después de 1996 & 51.7 & Después de 1996 & 43.3 & \\
\hline Toma de TAR & Toma & 100 & Toma & $\begin{array}{c}10 \\
0\end{array}$ & $\mathrm{~F}>.05$ \\
\hline & No toma & 0 & No toma & 0 & \\
\hline Carga viral & $\begin{array}{l}\text { Indetectable } \\
\text { Detectable }\end{array}$ & $\begin{array}{l}90.0 \\
10.0\end{array}$ & $\begin{array}{l}\text { Indetectable } \\
\text { Detectable }\end{array}$ & $\begin{array}{r}96.7 \\
3.3\end{array}$ & $\mathrm{~F}>.05$ \\
\hline Linfocitos T- & Menor de 200 cél $/ \mu \mathrm{L}$ & 13.3 & $\begin{array}{l}\text { Menor de } 200 \\
\text { cél } / \mu \mathrm{L}\end{array}$ & 0 & $\begin{array}{l}X^{2} \leq \\
.05\end{array}$ \\
\hline CD4 & $\begin{array}{l}\text { Entre } 200 \text { y } 499 \\
\text { cél/uL }\end{array}$ & 48.3 & $\begin{array}{l}\text { Entre } 200 \text { y } 499 \\
\text { cél } / \mu \mathrm{L}\end{array}$ & 36.7 & \\
\hline & Mayor de 500 cél $/ \mu \mathrm{L}$ & 38.3 & $\begin{array}{l}\text { Mayor de } 500 \\
\text { cél } / \mu \mathrm{L}\end{array}$ & 63.3 & \\
\hline Año toma & $\bar{x}$ & 15.36 & & & \\
\hline TAR & Rango & $0.5-31$ & & & \\
\hline Nadir CD4 & $\begin{array}{l}\text { Menor de } 350 \text { cél } / \mu \mathrm{L} \\
\text { Mayor de } 350 \text { cél } / \mu \mathrm{L} \\
\text { No se acuerda }\end{array}$ & $\begin{array}{l}25.0 \\
28.3 \\
46.7\end{array}$ & & & \\
\hline $\begin{array}{l}\text { Lesión } \\
\text { neurológica }\end{array}$ & $\begin{array}{l}\text { No } \\
\mathrm{Si}\end{array}$ & $\begin{array}{l}50.0 \\
50.0\end{array}$ & & & \\
\hline Medicación & $\begin{array}{l}\mathrm{No} \\
\mathrm{Si}\end{array}$ & 28.3 & & & \\
\hline
\end{tabular}

Fuente: Elaboración propia.

Y con respecto al segundo objetivo, atendiendo a los principios teóricos de vulnerabilidad social propuestos por Sánchez-González y Egea-Jiménez (2011), en esta investigación se propone el término de PVIH en situación de vulnerabilidad social, definido como un colectivo dentro de la población VIH que no disponen 0 aún no cuentan con las capacidades o activos para afrontar los conflictos o dificultades que se les presentan en la vida cotidiana, causado por factores personales, coyunturales y estructurales, en el cual puede concurrir cualquier PVIH, precisando por todo ello de residir de forma temporal o permanente en un centro residencial.

\section{DISCUSIÓN}

Atendiendo al primero objetivo de este estudio, la tabla 3 muestra como los dos grupos de PVIH están equiparados en edad ( $p>.05)$, nacionalidad ( $F>.05)$, año de diagnóstico del VIH antes o después de 1996 ( $F>.05)$, detectabilidad de la carga viral ( $F>.05)$, participación en las tareas de hogar $(F>.05)$ y en la toma de TAR $(F>.05)$. Por el contrario, las PVIH que residen en centros residenciales muestran menores recuentos de linfocitos T-CD4 $\left(X^{2} \leq .05\right)$, son mayoritariamente hombres $(\mathrm{F} \leq .05)$, presentan un menor nivel de estudios $\left(\mathrm{X}^{2} \leq .05\right)$, la gran mayoría están desempleados $(\mathrm{F} \leq$ $.05)$, participan en menor medida en voluntariados $(F \leq .05)$ y presentan una mayor asistencia a cursos 0 clases $(F \leq .05)$.

Estas características sociodemográficas y clínicas, permiten afirmar que las PVIH que residen en centros residenciales conforman un colectivo dentro de la población general con VIH ya que, a pesar de compartir características básicas, muestran una serie de particularidades.

Con respecto a la literatura existente, entre las características sociales, se ve un gran desempleo en las PVIH que residen en centros residenciales (96.7\%), lo cual concuerda con aquellos autores que hablan de las dificultades económicas que sufren las PVIH en general (Fuster-RuizdeApodaca et 
al., 2018a) y las PVIH que residen en centros residenciales (Peña y Álvarez, 1998; CCASBU, 2017). Con respecto al consumo de sustancias, el $78.3 \%$ de las $\mathrm{PVIH}$ que residen en centros residenciales han tenido un abuso de sustancias que han afectado a su vida, lo cual concuerda con lo descrito por autores como Jaquotot et al. (2002). Recordar que el 71.7\% tomaban medicación prescrita por un psiquiatra, lo cual concuerda con aquellos autores que hablan que las PVIH en centros residenciales presentan problemas de salud mental (Jaquotot et al., 2002; Vergara-Moragues et al., 2003; CCASBU, 2017).

Y en relación a las características clínicas, destacar que casi la mitad son PVIH que llevan viviendo con el VIH al menos 22 años al ser diagnosticadas antes de 1996, pudiéndose decir que casi la mitad de las PVIH que residen en centros residenciales son personas sobrevivientes del VIH a largo plazo, definidas como aquellas personas que llevan más de 25 años diagnosticadas de infección por VIH (Anile et al., 2018). El 90\% presentan una carga viral indetectable, cumpliendo este colectivo de PVIH con el tercer 90 que promulga ONUSIDA (UNAIDS, 2014), por el que en el 2020 el 90\% de las PVIH diagnosticadas con TAR deben de alcanzar una carga viral indetectable. Además muestran bajos recuentos de linfocitos T-CD4, lo cual concuerda con los informes técnicos de ONGs, que exponen que las PVIH en centros residenciales se encuentran en fases avanzadas de la infección por VIH (CCASBU, 2017). Y finalmente se destaca que casi la mitad de las PVIH que residen en centros residenciales que recordaban su Nadir CD4, presentaban un Nadir CD4 menor de 350 cél/ $\mu \mathrm{L}$ o diagnóstico tardío del VIH, lo cual coincide con los informes técnicos que hablan que las PVIH en centros residenciales pueden presentar un diagnóstico tardío del VIH (CCASBU, 2017). Y respecto al segundo objetivo, y partiendo de que las PVIH que residen en centros residenciales han sido poco estudiadas en la literatura científica, se propone el término de PVIH en situación de vulnerabilidad social con el fin de describir el sitio que ocupa este colectivo en nuestra sociedad, destacando la potencialidad y la capacidad de aprendizaje y mejora del mismo, alejándonos de aquellas concepciones meramente clasificatorias e incluso estigmatizadoras.

\section{CONCLUSIONES}

Las PVIH que residen en centros residenciales son un colectivo dentro de la población VIH con una serie de características sociodemográficas y clínicas propias.

Se propone el término de PVIH en situación de vulnerabilidad social para referirse a este colectivo, el cual denota su capacidad de aprendizaje, que es el punto de partida para el desarrollo de intervenciones socioeducativas que mejoren su CdV.

\section{REFERENCIAS BIBLIOGRÁFICA}

Anile, L., Marino, A., Gussio, M., Locatelli, M. E., Pampaloni, A., Vinci, L.,... y Celesi, B. M. (2018, Octubre). Long-term survivors in a cohort of HIV+ patients diagnosed between 1985 and 1992: predictive factors associated with more than 25 years of survival. Presentado en el HIV Drug Therapy, Gasgow. Resumen. Recuperado de http://www.natap.org/2018/GLASGOW/GLASGOW 65.htm.

Área de Vigilancia de VIH y Comportamientos de Riesgo (2018). Vigilancia Epidemiológica del VIH y sida en España 2017: Sistema de Información sobre Nuevos Diagnósticos de VIH y Registro Nacional de Casos de Sida. Plan Nacional sobre el Sida - D.G. de Salud Pública, Calidad e Innovación / Centro Nacional de Epidemiología - ISCIII. Madrid. Recuperado de http://www.isciii.es/ISCIII/es/contenidos/fd-servicios-cientifico-tecnicos/fd-vigilanciasalertas/fd- enfermedades/fd-sida/pdf sida/Informe VIH SIDA 2018 21112018.pdf

Blackstone, K., ludicello, J. E., Morgan, E. E., Weber, E., Moore, D. J., Franklin, D. R.,... y Woods, 


\section{CONCEPTUALIZACIÓN Y CARACTERÍSTICAS SOCIODEMOGRÁFICAS/CLÍNICAS DE LAS PERSONAS CON VIH QUE RESIDEN EN CENTROS RESIDENCIALES}

S. P. (2013). HIV infection Heightens Concurrent Risk of Functional Dependence in Persons With Chronic Methamphetamine Use. Journal of Addiction Medicine, 7(4), 255-263. doi:10.1097/ADM.0b013e318293653d.

Comité Ciudadano AntiSida de Burgos (CCASBU). (2017). Casa de Acogida la Encina, Memoria Técnica 2017.

Deeks, S. G. (2011). HIV infection, Inflammation, Immunosenescence, and Aging.

Annual Review Medicine, 62, 141-155. doi:10.1146/annurev-med-042909-093756. Erlandson, K. M., Allshouse, A. A., Jankowski, C. M, Duong, S., Mawhinney, S.,

Kohrt, W. M. y Campbell, T. B. (2012). Comparison of functional status instruments in HIV-infected adults on effective antiretroviral therapy. HIV Clinical Trials, 13(6), 324-34. doi:10.1310/hct1306-324.

Fuster-RuizdeApodaca, M. J. (2018b). Cuarto 90: Calidad de vida de las personas con VIH. Revista Multidisciplinar del Sida, 6 (14), 26-27. Recuperado de http://www.revistamultidisciplinardelsida.com/cuarto- 90-calidad-de-vida-de-las-personas-con-vih/

Fuster-RuizdeApodaca, M. J., Molero, F., Holgado, F. P. y Ubillos, S. (2015). Adaptation of the HIV Stigma Scale in Spaniards with HIV. Spanish Journal of Psychology, 18, E66. doi:10.1017/S1138741615000694.

Guaraldi, G., Orlando, G., Zona, S., Menozzi, M., Carli, F., Garlassi, E.,... y Palella, F. (2011). Premature age-related comorbidities among HIV-infected persons compared with the general population. Clinical Infectious Diseases, 53(11), 1120- 1126. doi:10.1093/cid/cir627.

Instituto Nacional de Estadística. (2017). Encuesta sobre centros y servicios de atención a personas sin hogar, Año 2016. Recuperado de https://www.ine.es/prensa/ecapsh_2016.pdf. Jaquotot, J.M.K., Valero, J. A., Ruiz, C. M., Zamazola, P. S. H., Ortega, F. P. y Cuesta, F. L. (2002). Tratamiento de la infección por VIH en pacientes con problemática psicosocial. Enfermedades Infecciosas y Microbiología Clínica, 20 (Supl 2), 19-28. Recuperado de http://www.elsevier.es/es-revista-enfermedades-infecciosas-microbiologia-clinica-28-articulotratamiento-infeccion-por-vih- pacientes-13037344

Kass, N. E., Munoz, A., Chen, B., Zucconi, S. L. y Bing, E. G. (1994). Changes in employment, insurance, and income in relation to HIV status and disease progression. The Multicenter AIDS Cohort Study. Journal of Acquired Immune Deficiency Syndromes, 7 (1), 86-91. Recuperado de https://www.ncbi.nlm.nih.gov/pubmed/8263757.

Miners, A., Phillips, A., Kreif, N., Rodger, A., Speakman, A., Fisher, M.,... y Lampe, F.

C. (2014). Health-related quality-of-life of people with HIV in the era of combination antiretroviral treatment: a cross-sectional comparison with the general population. The lancet HIV, 1(1), e32e40. doi:https://doi.org/10.1016/S2352- 3018(14)70018-98.

Peña, R. y Álvarez, R. G. (1998). Programa de atención integral a afectados por VIH/SIDA. Documentos de Trabajo Social: Revista de Trabajo Social y Acción Social, 12, 7-28. Recuperado de http://www.trabajosocialmalaga.org/archivos/revista_dts_numeros/DTS_12.pdf.

Sánchez-González, D. y Egea-Jiménez, C. (2011). Enfoque de vulnerabilidad social para investigar las desventajas socioambientales. Su aplicación en el estudio de los adultos mayores. Papeles de población, 17(69). Recuperado de http://www.scielo.org.mx/scielo.php?script=sci_arttext\&pid=S1405-

Servicio de Epidemiología de la Junta de Castilla y León. (2017). Vigilancia epidemiológica de nuevas infecciones por VIH Castilla y León. 2017. Recuperado de https://www.saludcastillayleon.es/ciudadanos/es/enfermedades-problemas-salud/vih-sidainfecciones-transmision-sexual. 
UNAIDS (2014). Ambitious Treatment Targets: Writing the final chapter of the AIDS epidemia. Recuperado de http://www.unaids.org/sites/default/files/media asset /JC2670 UNAIDS Treatment Targets en.pdf

UNAIDS (2018). Data 2018. Recuperado de http://www.unaids.org/sites/default/files/ media asset/unaids- data-2018 en.pdf

Vergara-Moragues, E., de Campos, A. V. y Girón-González, J. A. (2003). Las casas de acogida para enfermos de SIDA. Su utilidad en la época del tratamiento antirretroviral de gran actividad. En J. P. Diaz, E. P. de la Llave y A. R. Román (Eds), La infección por el VIH. Guía práctica (pp. 577586). Sevilla, España: Sociedad Andaluza de Enfermedades Infecciosas. 
